\title{
ЭКСИЛАМПЫ КАК ПЕРСПЕКТИВНЫЙ ИСТОЧНИК УФ ИЗЛУЧЕНИЯ ДЛЯ ФОТОХИМИЧЕСКОГО ОБЕЗВРЕЖИВАНИЯ МЫШЬЯКСОДЕРЖАЩИХ ВОД
}

\author{
Цыбикова Бэлэгма Амоголоновна', \\ belegmats@mail.ru \\ Батоева Агния Александровна', \\ abat@binm.ru \\ ' Байкальский институт природопользования СО РАН,
Россия, 670047, г. Улан-Удэ, ул. Сахьяновой, 6.
}

Актуальность исследования обусловлена необходимостью решения проблемы поступления в водные объекты токсичных веществ, в том числе соединений мышьяка, путем поиска и разработки эффективных фотохимических методов очистки и обезвреживания мышьяксодержащих растворов с использованием экологически чистых окислителей и современных безртутных источников УФ-излучения.

Цель: изучить возможности использования эксиламп для обезвреживания Аs-содержащих вод и установить закономерности фотохимической активации окисления мышьяка пероксидом водорода с использованием безртутного источника квазимонохроматического излучения $\mathrm{KrCl}-$ эксилампы.

Объекты: модельные водные растворы мышьяка (III) с концентрацией 2,7 мкмоль•лㄱ ( 0,2 мг/л), приготовленные на дистиллированной (УЭП=2 мкСм/см, рН 5,8) и водопроводной воде.

Методы: спектрофотометрическое определение ионов мышьяка (III); контроль рН потенциометрическим методом, съемка электронных спектров поглощения растворов реагирующих веществ; поглощенная мощность излучения эксилампы, определенная методом химической актинометрии с атразином; оценка доз УФ-излучения.

Результаты. Установлена принципиальная возможность использования безртутного источника квазимонохроматического излучения $\mathrm{KrCl}$-эксилампы (222 нм) для фотохимического окисления $\mathrm{As}(\mathrm{III})$ комбинированной системой $\left\{У Ф / \mathrm{H}_{2} \mathrm{O}_{2}\right\}$. Изучены основные закономерности фотохимического окисления мышьяка (III) с использованием безртутного источника УФ-излучения KrCl-эксилампы (222 нм). Проведены сравнительные исследования эффективности прямого фотолиза и комбинированного окисления мышьяка (III) в водном растворе ультрафиолетовым излучением KrCl-эксилампы в присутствии пероксида водорода. По эффективности окисления Аs(III) рассмотренные окислительные системы можно выстроить в ряд: $\left\{У Ф / H_{2} \mathrm{O}_{2}\right\}>\left\{\mathrm{H}_{2} \mathrm{O}_{2}\right\}>\{У Ф\}$. При комбинированной обработке $\left\{У Ф / \mathrm{H}_{2} \mathrm{O}_{2}\right\}$ наблюдается существенная интенсификация процесса окисления, через 10 минут остаточная концентрация мышьяка в растворе - ниже ПДК (0,01 мг/л). Также рассмотрено влияние гидрокарбонат-ионов, наиболее характерных анионов природных «водных матриц», на скорость реакции окисления As(III). Предложен ион-радикальный механизм фотохимического окисления мышьяка в комбинированной окислительной системе $\left\{\right.$ У $\left./ \mathrm{H}_{2} \mathrm{O}_{2}\right\}$ с использованием УФизлучения $\mathrm{KrCl}$ эксилампы.

\section{Ключевые слова:}

Мышьяк, пероксид водорода, безртутный источник УФ-излучения, KrCl-эксилампа, гидрокарбонаты, комбинированные окислительные процессы.

\section{Введение}

В последнее десятилетие мировое сообщество обеспокоено проблемой поступления в водные объекты токсичных веществ, в том числе соединений мышьяка $[1,2]$. Поступление данного загрязнителей в окружающую среду обусловлено как естественными (выветривание минералов и геотермальные процессы) [3-5], так и антропогенными факторами (сельское хозяйство, электроника, производство красителей, добыча и переработка упорных золото-, мышьяксодержащих руд). Учитывая опасные последствия для организма, которые, в зависимости от уровня воздействия, могут выражаться в кожных поражениях, периферической невропатии, диабете, сердечно-сосудистых и онкологических заболеваниях [6], очевидна необходимость глубокой очистки токсичных мышьяксодержащих вод.

Для очистки природных, сточных вод и технологических растворов от мышьяка применяется множество методов, наиболее широко известными из которых являются методы адсорбции/соосаждения и окислительные методы [7-9].

В качестве адсорбционных материалов широко применяют активированные угли, смолы, гели, сорбенты на основе $\mathrm{Si}$, модифицированные гидроксидами металлов $\left(\mathrm{Fe}^{2+}, \mathrm{Fe}^{3+}\right), \mathrm{TiO}_{2}, \mathrm{Fe}^{0}(Z V I)$, а также шлаки, угли, полученные из сельскохозяйственных отходов, биосорбенты, гетиты и др. $[7,10,11]$. Основными достоинствами сорбционных методов являются: доступность и относительная дешевизна сорбентов, простота технологических схем процессов. Однако они не лишены недостатков, главными из которых являются: ограниченная сорбционная емкость сорбентов, требующая многократного разбавления высококонцентрированных растворов, необходимость дальнейшей регенерации сорбентов и дополнительного обезвреживания и утилизации большого количества образующихся высокотоксичных и высококонцентрированных элюатов. А также проблема по утилизации отработанного сорбционного материала. 
Из окислительных методов для обезвреживания различных As-содержащих растворов чаще всего применяются те, в которых в качестве реагента окислителя используют оксид марганца $\left(\mathrm{MnO}_{4}^{-}\right)$, соединения «активного» хлора $\left(\mathrm{Cl}_{2}\right.$ или $\left.\mathrm{OCl}^{-}\right)$, основными недостатками которых являются: токсичность самого реагента и повышение солесодержания очищенной воды. Более безопасны, на наш взгляд, методы с применением экологически безопасных окислителей - кислорода воздуха и пероксида водорода (прежде всего вследствие отсутствия вторичного загрязнения воды продуктами восстановления реагентов). При этом методы обладают существенным недостатком, к которому относится высокая длительность процесса обработки. Так, период полупревращения процесса окисления As (III) кислородом достигает от 1 до 3 лет [12], а медленное окисление мышьяка под действием пероксида водорода [13] даже при многократном избытке окислителя свидетельствует в пользу применения комбинированных окислительных процессов, так называемых «Advanced Oxidation Processes» (AOP) [14-16]. Суть AOP процессов заключается в жидкофазном цепном окислении загрязняющих веществ (поллютантов) генерированными in situ высокореакционноспособными активными формами кислорода (АФК) - гидроксильными радикалами, синглетным кислородом и другими. Среди АОР методов, интенсивно развивающихся в последнее время, наиболее перспективны фотоактивированные окислительные процессы [17].

В технологиях очистки и обезвреживания воды в качестве источников УФ излучения традиционно используются ртутные газоразрядные лампы разного давления, основным и весомым недостатком которых является высокое содержание металлической ртути (от 10 мг в лампах низкого давления и до 600 мг в лампах высокого давления) [16, 17]. В настоящее время все большее распространение получает новый класс современных безртутных источников УФ излучения (газоразрядные эксимерные и эксиплексные лампы (эксилампы), $L E D$ лампы и др.) [18]. Главные достоинства эксиламп заключаются в том, что до $80 \%$ и более общей мощности излучения сосредоточено в узкой полосе излучения [19], они отличаются высокой энергией фотона $(3,5-10$ эВ) и не содержат ртути. Известны исследования по комбинированной деградации токсичных хлорорганических загрязнителей УФ излучением $\mathrm{KrCl}-(222 \mathrm{Hм})$ и $\mathrm{XeBr}-(283 \mathrm{нм})$ эксиламп в присутствии пероксида водорода, свидетельствующие о высокой эффективности окислительных процессов с использованием эксиламп по сравнению с аналогичными процессами с использованием ртутных ламп, вследствие значительного повышения интенсивности генерации АФК [20-22]. Известны немногочисленные исследования по применению ультрафиолетового облучения (УФ) в сочетании с окислителями для обезврежи- вания поллютантов неорганической природы $[17,23]$, в том числе Аs-содержащих растворов [24].

Цель данной работы: исследовать возможности использования эксиламп для обезвреживания мышьяксодержащих вод и установить закономерности фотохимической активации окисления мышьяка пероксидом водорода с использованием современного безртутного источника квазимонохроматического излучения $\mathrm{KrCl}$-эксилампы

\section{Материалы и методика эксперимента}

Объектом исследования являлись модельные водные растворы мышьяка (III) с концентрацией 2,7 мкмоль• л $^{-1}(\sim 0,2$ мг/л), приготовленные на дистиллированной (УЭП=2 мкСм/см, $\mathrm{pH} 5,8)$ и водопроводной воде (табл. 1). Содержание ионов мышьяка (III) контролировали спектрофотометрическим методом с молибдатом аммония [25]. Величину $\mathrm{pH}$ контролировали на иономере pH-150 M. Электронные спектры поглощения водных растворов реагирующих веществ снимали на спектрофотометре UV-VIS Agilent 8453. В работе использовали реактивы: $\mathrm{Na}_{3} \mathrm{AsO}_{3}$ (ч.д.а), $\mathrm{H}_{2} \mathrm{O}_{2}$ (30\%-й раствор), 3АО «Химреактивснаб». Окислитель $\mathrm{H}_{2} \mathrm{O}_{2}$ вводили в реакционную смесь единовременно, при стехиометрическом молярном соотношении $\left[\mathrm{H}_{2} \mathrm{O}_{2}\right]-\left[\mathrm{As}^{3+}\right]$, равном 1. Для корректировки $\mathrm{pH}$ растворов использовали $0,1 \mathrm{~N}$ растворы $\mathrm{NaOH}$ или $\mathrm{H}_{2} \mathrm{SO}_{4}$.

Таблица 1. Характеристика водопроводной воды

Table 1. Characteristics of tap water

\begin{tabular}{|l|c|}
\hline \multicolumn{1}{|c|}{ Показатель/Characteristics } & Значение/Value \\
\hline $\mathrm{pH}$ & $7,10 \pm 0,2$ \\
\hline $\begin{array}{l}\text { Удельная электрическая проводимость, мкСм/см } \\
\text { Specific electrical conductivity, } \mu \mathrm{S} / \mathrm{cm}\end{array}$ & $236 \pm 7$ \\
\hline $\begin{array}{l}\text { Общий органический углерод, мг/л } \\
\text { Total organic carbon, mg/L }\end{array}$ & $3,3 \pm 0,9$ \\
\hline Жесткость, мг-экв/л/Stiffness, meq/L & $2,60 \pm 0,39$ \\
\hline Гидрокарбонаты, мг/л/Hydrocarbonates, mg/L & $134 \pm 16$ \\
\hline Аммоний, мг/л/Ammonium, mg/L & $<0,05$ \\
\hline Нитриты, мг/л/Nitrite, mg/L & $<0,1$ \\
\hline Нитраты, мг/л/Nitrates, mg/L & $4,81 \pm 0,63$ \\
\hline Натрий, мг/л/Sodium, mg/L & $9,32 \pm 1,59$ \\
\hline Магний, мг/л/Magnesium, mg/L & $9,73 \pm 0,20$ \\
\hline Кальций, мг/л/Calcium, mg/L & $36,07 \pm 0,76$ \\
\hline Хлориды, мг/л/Chlorides, mg/L & $0,30 \pm 0,04$ \\
\hline Сульфаты, мг/л/Sulphates, mg/L & $16,58 \pm 2,16$ \\
\hline Железо (II), мг/л/Iron (II), mg/L & н/o/not detected \\
\hline
\end{tabular}

Исследования основных кинетических закономерностей фотохимического окисления As(III) проводили на лабораторной установке, которая включала последовательно расположенные по направлению движения обрабатываемой воды термостатированную емкость (усреднитель), перистальтический насос и проточный фотореактор, состоящий из трех последовательно соединенных кварцевых трубок (внешний диаметр 14 мм, внутрен- 
ний диаметр 11 мм, длина трубки 100 мм), максимально приближенных к УФ-источнику ( $\mathrm{h}=5 \mathrm{mм})$, и отражателя, выполненного из алюминиевого листа (рис. 1). Объем обрабатываемого раствора составлял 0,3 л, скорость циркуляции раствора 0,5 л/мин, температура $25 \pm 2{ }^{\circ} \mathrm{C}$. Источник УФ-из-

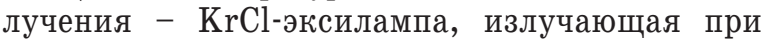
222 нм, с полушириной спектральной полосы 2-15 нм (000 «Эксилампы», Институт сильноточной электроники СО РАН, г. Томск). Поглощенная мощность излучения эксилампы, определенная методом химической актинометрии с атразином [20], составила $0,55 \mathrm{mBт} / \mathrm{cm}^{2}$. Дозы УФ-излучения определяли как произведение продолжительности облучения и интенсивности поглощенного излучения в объеме, рассчитанной с использованием интегрированной формы закона Бугера-Ламберта-Бера [26]. Потребляемую мощность электрической энергии выбранного источника контролировали с помощью измерителя Voltcraft EM-3000.

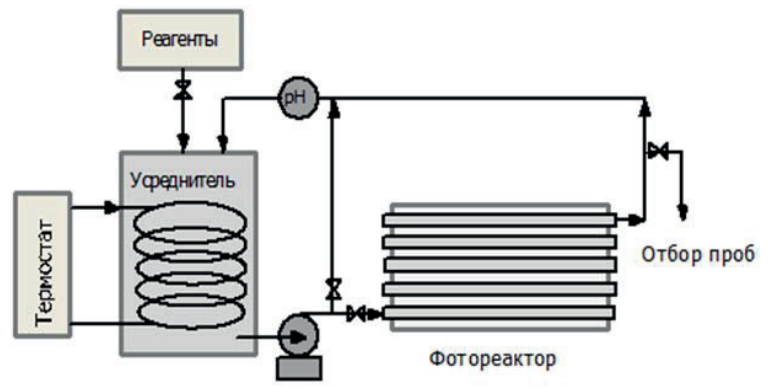

Рис. 1. Схема экспериментальной установки

Fig. 1. Schematic representation of the experimental setup

\section{Результаты и их обсуждение}

Предварительно оценена фотохимическая активность As(III) при прямом фотолизе. Известно, что в результате протекания первичных фотохимических процессов (фотодиссоциации, фотоактивации, внутримолекулярного окисления и фотовосстановления) при прямом фотолизе в системе генерируются высокоактивные частицы с неспаренным электроном - радикалы, участвующие во вторичных процессах окисления и деструкции загрязнителей:

$$
\begin{gathered}
\mathrm{H}_{2} \mathrm{O}+h v \rightarrow \cdot \mathrm{OH}+\mathrm{H}^{+} \\
\mathrm{HO}+{ }^{\cdot} \mathrm{OH} \rightarrow \mathrm{H}_{2} \mathrm{O}_{2}
\end{gathered}
$$

Необходимо отметить, что скорость генерирования $\mathrm{OH}$ радикалов определяется в основном свойствами источника УФ-излучения и присутствием реагентов - окислителей, являющихся прекурсорами АФК. Так, характер кинетической кривой прямого фотолиза при облучении $\mathrm{KrCl}$-эксилампой (рис. 2) по сравнению с кинетической кривой, полученной с ртутной УФ-С лампой (254 нм) в аналогичных условиях [27], значительно отличается: процесс окисления As(III) под действием эксилам- пы почти в 20 раз быстрее (начальные скорости реакции окисления As(III), рассчитанные по уравнению $W_{0}=d C_{\mathrm{As}}{ }^{3+} / d t$, составили $0,80 \mathrm{м \kappa моль} \cdot \mathrm{J}^{-1} \cdot \mathrm{мин}^{-1}$ и 0,04 мкмоль $Л^{-1} \cdot$ мин $^{-1}$, соответственно), а эффективность процесса через 5 минут обработки достигает 36,5 \% по сравнению с 9 \% при УФ-С фотолизе. При этом стоит отметить, что, несмотря на высокую степень окисления, дальнейшее увеличение продолжительности УФ обработки не приводит к существенному росту әффективности процесса, через 30 минут эффективность составила лишь $43 \%$, что обуславливает целесообразность применения комбинированной окислительной системы.

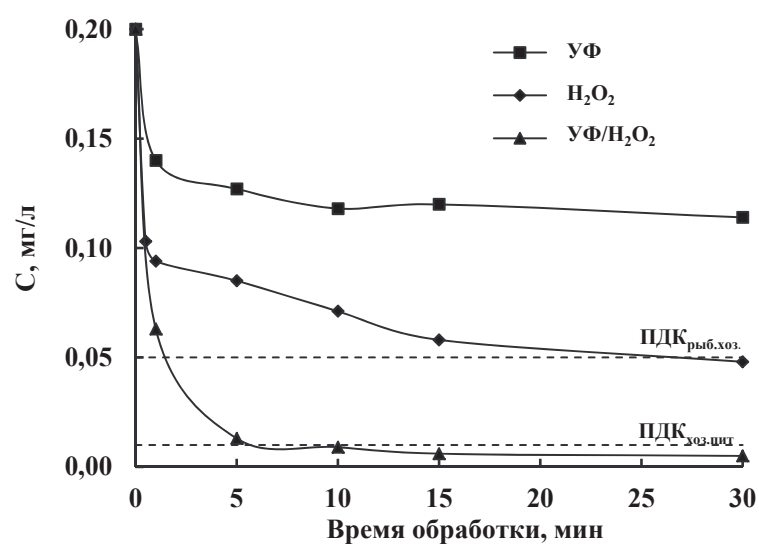

Puс. 2. Сравнительные эксперименты по окислению As(III). $\left[\mathrm{As}^{3+}\right]_{0}=2,7$ мклоль $/ л,\left[\mathrm{H}_{2} \mathrm{O}_{2}\right]=2,7$ мклоль $/ л, \mathrm{pH} 5,8$

Fig. 2. Comparative experiments on As (III) oxidation. $\left[\mathrm{As}^{3+}\right]_{0}=2,7 \mu \mathrm{M},\left[\mathrm{H}_{2} \mathrm{O}_{2}\right]=2,7 \mu \mathrm{M}, \mathrm{pH} 5,8$

При этом следует отметить, что при окислении As(III) в темновом процессе («dark»), описываемом брутто-реакцией [15]:

$$
\mathrm{As}(\mathrm{OH})_{3}+\mathrm{H}_{2} \mathrm{O} \Rightarrow \mathrm{HAsO}_{4}{ }^{2-}+2 \mathrm{H}^{+}+\mathrm{H}_{2} \mathrm{O}
$$

несмотря на достаточно высокую начальную скорость окисления, равную 1,41 мкмоль $\cdot{ }^{-1} \cdot \mathrm{мин}^{-1}$, наблюдалась дезактивация реакционной смеси в течении первых 15 минут, а дальнейшая обработка не приводила к значимому увеличению эффективности процесса (76 \% через 30 минут обработки).

При облучении растворов As(III) в присутствии пероксида водорода в комбинированной окисли-

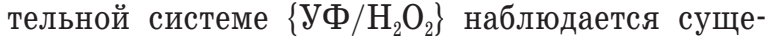
ственная интенсификация процесса окисления $\mathrm{As}(\mathrm{III})$, начальная скорость окисления увеличилась в 2,3 раза по сравнению с прямым фотолизом и составила 1,82 мкмоль $\pi^{-1} \cdot \mathrm{MиH}^{-1}$. Важно отметить, что в комбинированной системе $\left\{\mathrm{У} / \mathrm{H}_{2} \mathrm{O}_{2}\right\}$ уже через 10 мин экспозиции остаточная концентрация As(III) в растворе составила 0,009 мг/л, что соответствует нормам ПДК $(0,01$ мг/л) [28, 29]. По эффективности, скорости процесса окисления As(III) рассмотренные окислительные системы можно выстроить в ряд: $\left\{\mathrm{У} \Phi / \mathrm{H}_{2} \mathrm{O}_{2}\right\}>\left\{\mathrm{H}_{2} \mathrm{O}_{2}\right\}>\{\mathrm{У} \Phi\}$.

Дана сравнительная оценка удельной энергоэффективности процессов фотохимического окисле- 
ния As(III) в различных комбинированных системах с использованием разных источников излучения (полученных результатов в сравнении с литературными данными) (табл. 2) по количеству окисленного вещества в расчете на единицу затраченной электрической энергии путем расчета удельной конверсии (Y) по представленной формуле:

$\left(\left(C_{0}-C_{t}\right) \times V \times 1000\right) /(W \times \tau \times 60)$, мкг $/ \kappa Д ж$,

где $C_{0}$ - начальная концентрация мышьяка (III), мкг/л; $C$ - концентрация мышьяка (III) после обработки, мкг/л; $V$ - объем обрабатываемого раствора, мл; $W$ - потребляемая мощность, Вт; $\tau-$ продолжительность процесса, мин.

Таблица 2. Сравнение различных источников УФ излучения при окислении As(III)

Table 2. Comparison of various sources of UV radiation during As(III) oxidation

\begin{tabular}{|c|c|c|c|c|c|}
\hline $\begin{array}{l}\text { Окислительная система } \\
\text { Oxidizing system }\end{array}$ & $\begin{array}{l}3 \\
2\end{array}$ & 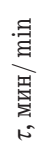 & $\begin{array}{c}\text { Эффективность } \\
\text { окисления } \\
\text { 0xidation } \\
\text { efficiency } \Psi, \%\end{array}$ & 置 & 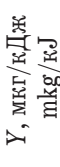 \\
\hline US/UV-254 [27] & 48 & 60 & 95,5 & 0,04 & 1,67 \\
\hline $\mathrm{UV}-\mathrm{Xe} / \mathrm{TiO}_{2}[30]$ & 300 & 150 & 80 & 2,90 & 1,0 \\
\hline UV-Xe/ $/ \mathrm{TiO}_{2} / \mathrm{Ag} /$ air [30] & 300 & 60 & 80 & 6,67 & 2,47 \\
\hline UV-222/ $\mathrm{H}_{2} \mathrm{O}_{2}{ }^{*}$ & 30 & 10 & 95,5 & 1,82 & 3,20 \\
\hline
\end{tabular}

При использовании широкополосного источника - ксеноновой лампы - в комбинированных системах UV-Xe/ $/ \mathrm{TiO}_{2} / \mathrm{Ag} /$ air и UV-Xe/ $/ \mathrm{TiO}_{2}$, несмотря на высокие значения начальных скоростей фотоокисления As (III) при значительной продолжительности обработки 60 и 150 мин, соответственно, эффективность окисления достигает лишь $80 \%$. [30]. Окисление As(III) в комбинированной окислительной системе US/UV-254 с использованием ртутной лампы также менее эффективно, при этом через 60 минут обработки конверсия достигает 95,5\% [27]. Рассмотренная нами окислительная система UV-222/ $\mathrm{H}_{2} \mathrm{O}_{2}$ с применением безртут-

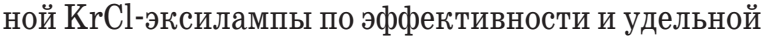
конверсии является предпочтительной.

По удельной конверсии рассмотренные комбинированные окислительные системы с различными источниками УФ излучения можно выстроить в ряд UV-222/ $\mathrm{H}_{2} \mathrm{O}_{2}>\mathrm{UV}-\mathrm{Xe} / \mathrm{TiO}_{2} / \mathrm{Ag} /$ air $>\mathrm{US} /$ $\mathrm{UV}-254 / \mathrm{H}_{2} \mathrm{O}_{2}>\mathrm{UV}-\mathrm{Xe} / \mathrm{TiO}_{2}$.

С точки зрения энергоэффективности и экологической безопасности оптимальным источником является безртутная $\mathrm{KrCl-эксилампа.} \mathrm{При} \mathrm{этом}$ важно отметить, что коэффициент молярной экстинкции пероксида водорода резко повышается в коротковолновой области спектра и составляет при 222 нм $1,12 \cdot 10^{5}$ л·моль ${ }^{-1} \cdot \mathrm{cm}^{-1}$, следовательно, его фотоактивация будет происходить быстрее, вследствие чего и скорость генерации гидроксильных радикалов будет выше (рис. 3 ).

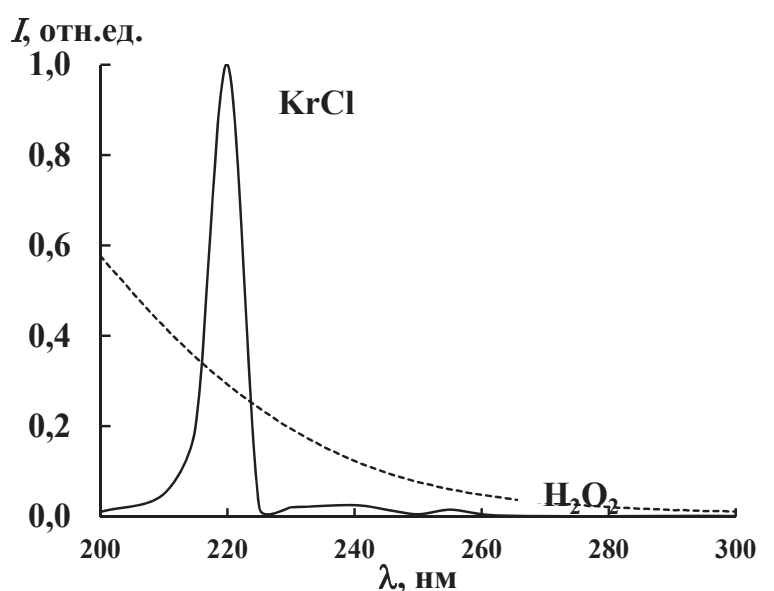

Рис.3. Спектры поглощения пероксида водорода и излучения KrCl-эксилампь

Fig. 3. Absorption spectra of hydrogen peroxide and $\mathrm{KrCl}$ excilamp radiation

Значительное увеличение скорости окисления $\mathrm{As}(\mathrm{III})$ в комбинированной окислительной системе $\left\{\mathrm{У} \Phi / \mathrm{H}_{2} \mathrm{O}_{2}\right\}$ является прямым следствием участия АФК и обусловлено фотоактивацией пероксида водорода с его диспропорционированием по известному радикально-цепному механизму Габера-Вейса с образованием неселективных высокореакционноспособных гидроксильных радикалов [31, 32]:

$$
\begin{gathered}
\mathrm{H}_{2} \mathrm{O}_{2}+h v \rightarrow 2 \cdot \mathrm{OH} \\
\mathrm{H}_{2} \mathrm{O}_{2}+\mathrm{OH} \rightarrow \mathrm{HO}_{2} \cdot+\mathrm{H}_{2} \mathrm{O} \\
k=3 \cdot 10^{7} \mathrm{л} \cdot \text { моль }^{-1} \mathrm{c}^{-1} \\
\mathrm{HO}_{2} \cdot+\mathrm{H}_{2} \mathrm{O}_{2} \rightarrow \cdot \mathrm{OH}+\mathrm{H}_{2} \mathrm{O}+\mathrm{O}_{2} \\
k=9,7 \cdot 10^{7} \text { л } \cdot \text { моль }^{-1} \mathrm{c}^{-1} \\
\left.\mathrm{HO}_{2} \text { (или } \cdot \mathrm{OH}\right)+\mathrm{HO}_{2} \cdot \rightarrow \mathrm{H}_{2} \mathrm{O}_{2}\left(\text { или } \mathrm{H}_{2} \mathrm{O}\right)+\mathrm{O}_{2} \\
k=8,3 \cdot 10^{5} \text { л } \cdot \text { моль }^{-1} \mathrm{c}^{-1}
\end{gathered}
$$

\section{Влияние pH среды на процесс окисления As (III)}

Показатель кислотности среды является одним из ключевых параметров при реализации фотоактивированных окислительных процессов. Результаты экспериментальной серии по обработке As-содержащих модельных растворов при различных значениях $\mathrm{pH}$ показали, что в кислой среде ( $\mathrm{pH} 3)$ при прямом фотолизе окисление As(III) протекает медленно, начальная скорость составила 0,068 мкмоль $\cdot \mathrm{J}^{-1} \cdot \mathrm{мин}^{-1}$, и при дозе УФ излучения, равной 1 Дж/ $\mathrm{cm}^{2}$, окисляется лишь $18 \%$ вещества. (рис. 4). При этом процесс в интервале 5,8-9 протекает быстрее в $(11,3-14,6)$ раза, значения начальных скоростей составляли $0,77-0,99$ мкмоль $\cdot \mathrm{\pi}^{-1} \cdot \mathrm{мин}^{-1}$, а доза УФ-из-

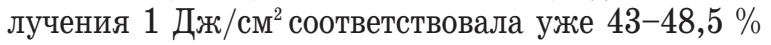
окисленного вещества. При этом в присутствии $\mathrm{H}_{2} \mathrm{O}_{2}$ процесс окисления $\mathrm{As}(\mathrm{III})$ значительно ускоряется при рН $3 W_{0}=1,77$ мкмоль ${ }^{-1} \cdot \mathrm{Mин}^{-1}$, а при $5,8-9,0-(1,85-2,17)$ мкмоль $\pi^{-1} \cdot$ мин $^{-1}$. Важно отметить, что при достаточно высокой скорости и эффективности комбинированного процесса (76 \%) при рН 3 наблюдали дезактивацию реакционной смеси уже в первые 5 мин, что объясняется влия- 

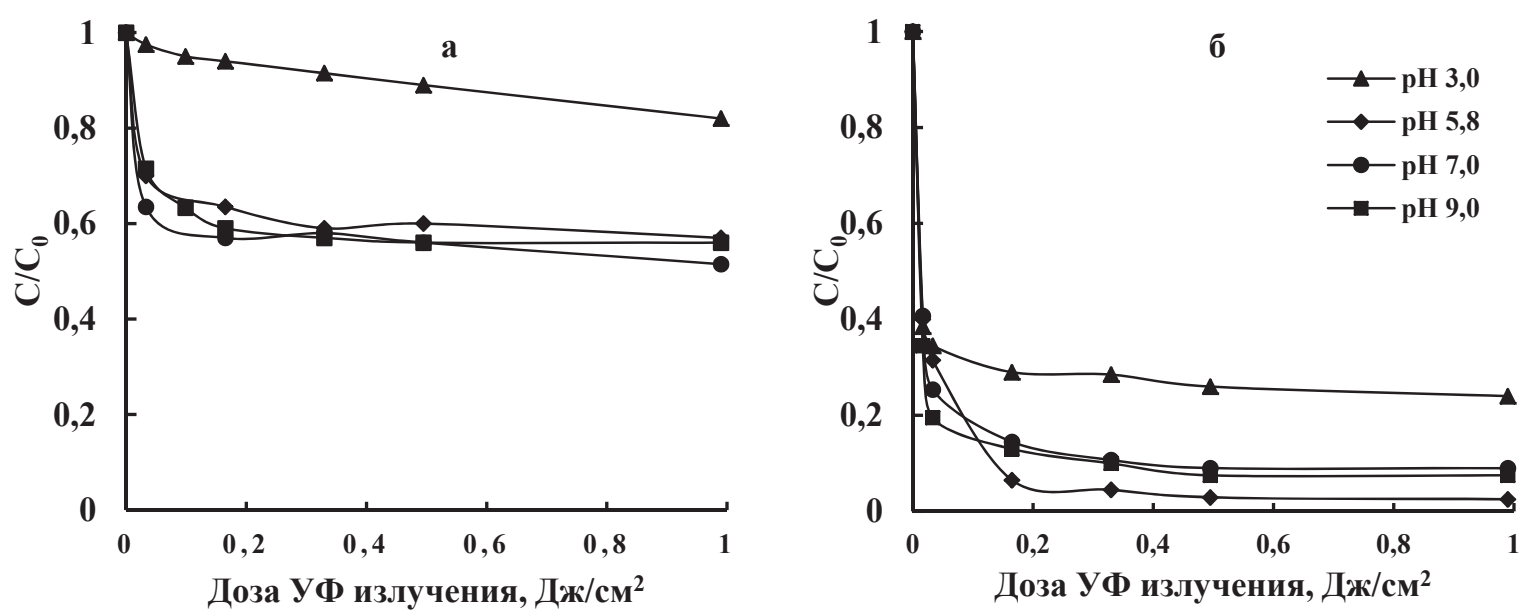

Pис.4. Влияние рН на процесс фотохимического окисления Аs (III) при прямом фотолизе $\{У \Phi\}$ (а) и в присутствии $\mathrm{H}_{2} \mathrm{O}_{2}$ $\left\{\mathrm{Y} \Phi / \mathrm{H}_{2} \mathrm{O}_{2}\right\}\left(\right.$ б). $\left[\mathrm{As}^{3+}\right]_{0}=2,7$ мккмоль $/ л,\left[\mathrm{H}_{2} \mathrm{O}_{2}\right]=2,7$ лкмоль $/ л$

Fig. 4. Effect of $\mathrm{pH}$ on the photochemical oxidation of $\mathrm{As}(\mathrm{III})$ in direct photolysis (UV) (a) and in the presence of $\mathrm{H}_{2} \mathrm{O}_{2}\left\{\mathrm{UV} / \mathrm{H}_{2} \mathrm{O}_{2}\right\}(\sigma)$. $\left[\mathrm{As}^{3+}\right]_{0}=2,7 \mu \mathrm{M},\left[\mathrm{H}_{2} \mathrm{O}_{2}\right]=2,7 \mu \mathrm{M}$

нием кислотности среды на скорость фоторазложения пероксида водорода и на процесс окисления различных интермедиатов. Кроме того, дозы УФизлучения, необходимые для окисления $90 \%$ $\mathrm{As}(\mathrm{III})$ в интервале $\mathrm{pH} \quad 5,8-9$ составляли 150-380 мДж/ $\mathrm{cm}^{2}$. Значительное сокращение доз УФ-излучения и ускорение процесса окисления $\mathrm{As}(\mathrm{III})$ является следствием участия АФК.

\section{Выявление роли АФК в процессе окисления As(III)}

Для подтверждения участия АФК в процессе фотоокисления As (III) проведены эксперименты с использованием радикальных ловушек - трет-бутанола и изо-пропанола. Константа взаимодействия трет-бутанола с $\mathrm{OH}$ радикалами составляет $6 \cdot 10^{8} \mathrm{~J} \cdot$ моль $^{-1} \cdot \mathrm{c}^{-1}$, а изо-пропанола $-1,9 \cdot 10^{9} \mathrm{~J} \cdot$ моль $^{-1} \cdot \mathrm{c}^{-1}$. Установлено, что введение добавок ингибирует процесс окисления $\mathrm{As}(\mathrm{III})$, через 30 минут обработки с изо-пропанолом эффективность процесса снизилась на 18,5\%, с трет-бутанолом - на $11 \%$ (в соответствии с рис. 5). Следовательно, при фотоокислении As(III) в рассматриваемой комбиниро-

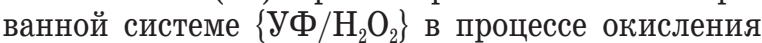
принимают участие гидроксильные радикалы, в соответствии с реакцией (7).

Вероятно, при фотоактивации пероксида водорода излучением эксилампы в данных экспериментальных условиях реализуется радикальный механизм окисления As(III), включающий процессы активации пероксида водорода УФ излучением с образованием гидроксильных радикалов (3).

Возможно также в рассматриваемой окислительной системе наряду с ' $\mathrm{OH}$ радикалами генерирование других АФК, в частности $\mathrm{HO}_{2}{ }^{\cdot-}$ и $\mathrm{O}_{2}{ }^{--}$, способных активировать пероксид водорода (5), а также рекомбинировать с образованием дополнительного количества пероксида водорода (6), фотоактивация которого увеличивает выход гидроксильных радикалов. Известно, что супероксидные ра- дикалы $\mathrm{O}_{2}{ }^{--}$являются наиболее долгоживущими частицами по сравнению с гидроксильными радикалами [32] и вследствие этого имеют более высокую степень растворения, чем гидроксильные радикалы, и в объёме реагируют с As(III) с образованием интермедиата As(IV), окисление которого и является лимитирующей стадией процесса. Известно, что интермедиаты As (IV), которые в зависимости от $\mathrm{pH}$ среды находятся в различных фор$\operatorname{Max}-\mathrm{As}^{\mathrm{IV}}(\mathrm{OH})_{4}, \mathrm{As}(\mathrm{OH})_{3} \mathrm{O}^{-}, \mathrm{HAs}^{\mathrm{IV}} \mathrm{O}_{3}^{-}, \mathrm{As}^{\mathrm{IV}} \mathrm{O}_{3}{ }^{2-}[24]$, далее окисляются: в кислой среде - с образованием двух продуктов, как конечного $\mathrm{As}(\mathrm{V})$, так и исходного As(III) (о чем свидетельствует дезактивация реакционной смеси при $\mathrm{pH} 3$ ), а в слабокислой и щелочной средах $(5,8-9,0)$ - до конечного $\mathrm{As}(\mathrm{V})$. Существование интермедиата As(IV) подтверждено методом импульсного радиолиза ранее авторами [33].

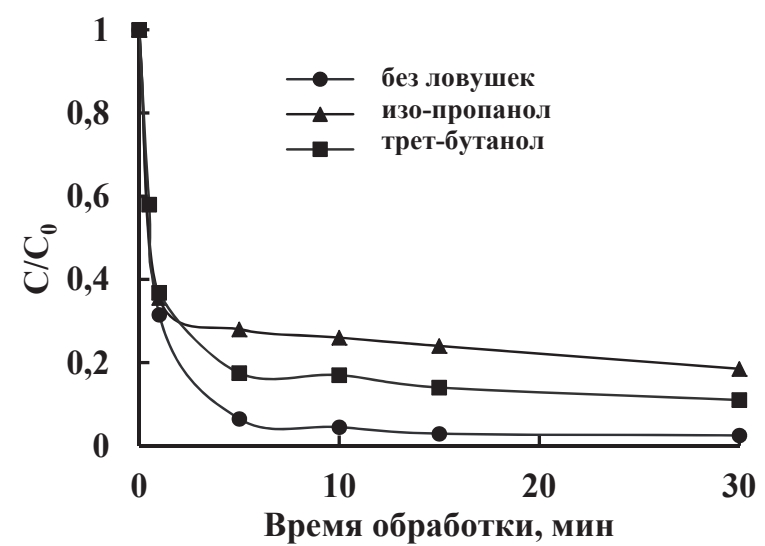

Puc. 5. Фотохимическое окисление As (III) в присутствии ловушек радикалов. $\left[\mathrm{As}^{3+}\right]_{0}=\left[\mathrm{H}_{2} \mathrm{O}_{2}\right]_{0}=2,7$ мкмоль $\boldsymbol{\Omega}^{-1}$, [uзо-пропанол] $=1,35$ млоль $/ л$, [трет-бутанол] $=1,35$ млоль $/ л$. pH 5,8

Fig. 5. Photochemical oxidation of As (III) in the presence of radical scavengers. $\left[\mathrm{As}^{3+}\right]_{0}=\left[\mathrm{H}_{2} \mathrm{O}_{2}\right]_{0}=2,7 \mu M$, [iso-propanol] $=1,35 \mathrm{mM}$, [tert-butanol] $=1,35 \mathrm{mM} . \mathrm{pH} 5,8$ 
Анализ литературы и полученные экспериментальные результаты позволяют предположить, что процесс фотохимического окисления мышьяка в комбинированной окислительной системе $\left\{\mathrm{У} \Phi / \mathrm{H}_{2} \mathrm{O}_{2}\right\}$ протекает по смешанному ион-радикальному механизму, включающему наряду с реакциями (1)-(6) следующие (7)-(15):

$$
\begin{aligned}
& \mathrm{As}(\mathrm{III})+{ }^{\cdot} \mathrm{OH} \rightarrow \mathrm{As}(\mathrm{IV})+\mathrm{OH}^{-} \\
& k=8,5 \cdot 10^{9} \mathrm{л} \cdot \text { моль }^{-1} \cdot \mathrm{c}^{-1} \\
& \mathrm{As}(\mathrm{III})+\mathrm{O}_{2} \cdot{ }^{--}+\mathrm{H}^{+} \rightarrow \mathrm{As}(\mathrm{IV})+\mathrm{HO}_{2}^{-}, \\
& k=3,6 \cdot 10^{6} \text { л } \cdot \text { моль }{ }^{-1} \cdot \mathrm{c}^{-1} \\
& 2 \mathrm{HAs}^{\mathrm{IV}} \mathrm{O}_{3}^{-} \leftrightarrow \mathrm{As}(\mathrm{III})+\mathrm{As}(\mathrm{V}) \\
& k=4,5 \cdot 10^{8} \pi \cdot \text { моль }^{-1} \mathrm{c}^{-1} \\
& \mathrm{As}^{\mathrm{IV}}(\mathrm{OH})_{4} \rightarrow \mathrm{As}(\mathrm{III})+\mathrm{As}(\mathrm{V}) \\
& k=8,4 \cdot 10^{8} \text { л } \cdot \text { моль }^{-1} \mathrm{c}^{-1} \\
& \mathrm{As}^{\mathrm{IV}}(\mathrm{OH})_{4}+\mathrm{HAs}^{\mathrm{IV}} \mathrm{O}_{3}^{-} \rightarrow \mathrm{As}(\mathrm{III})+\mathrm{As}(\mathrm{V}) \\
& k=2,0 \cdot 10^{9} \mathrm{л} \cdot \text { моль }^{-1} \mathrm{c}^{-1} \\
& \mathrm{As}^{\mathrm{IV}}(\mathrm{OH})_{4} \leftrightarrow \mathrm{As}(\mathrm{OH})_{3} \mathrm{O}^{-}+\mathrm{H}^{+} \\
& p K=7,26 \\
& \mathrm{HAs}^{\mathrm{IV}} \mathrm{O}_{3}{ }^{-} \leftrightarrow \mathrm{As}^{\mathrm{IV}} \mathrm{O}_{3}{ }^{2-}+\mathrm{H}^{+} \\
& p K=7,57 \\
& \mathrm{As}(\mathrm{OH})_{3} \mathrm{O}^{-}+\mathrm{O}_{2} \rightarrow \mathrm{As}(\mathrm{V})+\mathrm{O}_{2}^{-} \\
& k=1,1 \cdot 10^{9} \pi \cdot \text { моль }^{-1} \mathrm{c}^{-1} \\
& \mathrm{As}^{\mathrm{IV}}(\mathrm{OH})_{4}+\mathrm{O}_{2} \rightarrow \mathrm{As}(\mathrm{V})+\mathrm{HO}_{2} / \mathrm{O}_{2}^{-} \\
& k=1,4 \cdot 10^{9} \text { л } \cdot \text { моль }^{-1} \mathrm{c}^{-1}
\end{aligned}
$$

Влияние гидрокарбонатов на процесс окисления As (III)

Известно, что природная вода является многокомпонентной динамической системой, так называемой «водной матрицей», которая содержит в своем составе различные газы, органические и минеральные вещества, присутствие которых оказывает значительное влияние на процессы, протекающие в природных водных системах.

Наиболее часто в природных водах встречаются гидрокарбонаты, хлориды, сульфаты щелочноземельных и щелочных металлов, в меньшей мере нитраты, нитриты, силикаты, фториды, фосфаты и др. На рис. 6 представлены результаты по окислению As(III) в комбинированной системе $\left\{\mathrm{У} / \mathrm{H}_{2} \mathrm{O}_{2}\right\}$ в присутствии гидрокарбонатов $\left(\mathrm{HCO}_{3}{ }^{-}\right)$ в концентрационном диапазоне 0-1300 мг/л. Установлено, что при концентрациях до 130 мг/л гидрокарбонат-ионы оказывают ингибирующее действие на протекание реакций в $\left\{У \Phi / \mathrm{H}_{2} \mathrm{O}_{2}\right\}$, скорость процесса снизилась до 1,6 мкмоль ${ }^{-1} \cdot \mathrm{MuH}^{-1}$, а эффективность окисления As(III) на 30 \% . Полагаем, что ингибирующее влияние $\mathrm{HCO}_{3}^{-}$на процесс фотохимического окисления As(III) связано с нецелевым расходованием ОН-радикалов согласно реакции [34-36]:

$$
\begin{gathered}
\mathrm{HCO}_{3}{ }^{-}+\cdot \mathrm{OH} \rightarrow \mathrm{HCO}_{3}{ }^{-}+\mathrm{OH} \\
k=8,5 \cdot 10^{6} \mathrm{~J} \cdot \mathrm{моль}^{-1} \cdot \mathrm{c}^{-1}
\end{gathered}
$$

Однако при десятикратном увеличении концентрации $\mathrm{HCO}_{3}^{-}(1300 \mathrm{мг} / л) W_{0}$ увеличилась до 2,23 мкмоль $\pi^{-1} \cdot \mathrm{Mин}^{-1}$ и уже после 15 минут обработки наблюдалось полное окисление мышьяка (III).

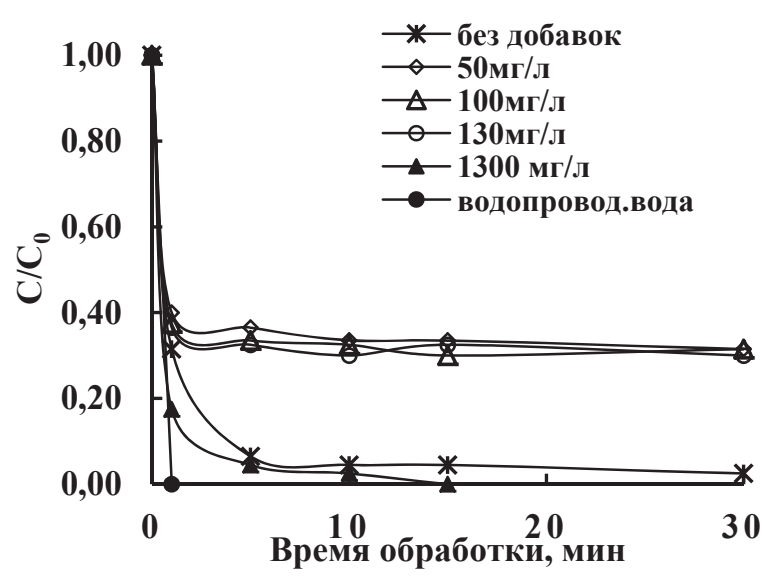

Pис. 6. Влияние гидрокарбонат-ионов на процессе фотохимического окисления $\mathrm{As}(\mathrm{III}) .\left[\mathrm{As}^{3+}\right]_{0}=\left[\mathrm{H}_{2} \mathrm{O}_{2}\right]_{0}=2,7$ мкмоль $/ л$

Fig. 6. Influence of hydrocarbonate ions on As (III) photochemical oxidation. $\left[\mathrm{As}^{3+}\right]_{0}=\left[\mathrm{H}_{2} \mathrm{O}_{2}\right]_{0}=2,7 \mu \mathrm{M} / \mathrm{L}$

Промотирующее влияние высоких концентраций $\mathrm{HCO}_{3}^{-}$и сокращение времени обработки обусловлено подщелачиванием обрабатываемого раствора ( $\mathrm{pH} \geq 9)$, созданием условий для ускорения процесса депротонирования $\mathrm{H}_{2} \mathrm{O}_{2}$. Неожиданные результаты получены при комбинированной $\left\{\mathrm{У} \Phi / \mathrm{H}_{2} \mathrm{O}_{2}\right\}$ обработке As-содержащего модельного раствора, приготовленного на водопроводной воде (табл. 1), скорость процесса окисления As(III) увеличилась в 1,5 раза, и уже в первую минуту достигается полное окисление «таргетного» загрязнителя. Вероятно, более эффективное окисление мышьяка в водопроводной воде обусловлено как протеканием фотохимических процессов в обрабатываемой воде с образованием различных АФК, фотоактивацией пероксида водорода, так и увеличением удельной электропроводности водопроводной воды вследствие содержания в ней различных солей, способствующих увеличению степени диспропорционирования пероксида водорода. Наряду с этим возможно участие растворенного органического вещества, которое способно фотоиндуцировать превращения экотоксикантов [37] в качестве фотосенсибилизатора.

\section{Выводы}

Изучены основные кинетические закономерности процесса фотохимического окисления токсичных соединений As (III) в комбинированной окислительной системе, базирующейся на использовании генерируемых in situ активных форм кислорода при воздействии монохроматического УФ-С излучения $\mathrm{KrCl}$-эксилампы.

Установлено, что при обработке в комбиниро-

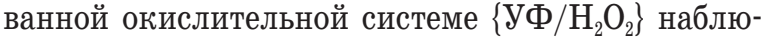
дается существенная интенсификация процесса окисления As(III), при этом уже через 10 минут остаточная концентрации загрязнителя в растворе ниже норм ПДК $(0,01$ мг/л). По эффективности, скорости процесса окисления As(III) рассмотренные окислительные системы можно выстроить в 
ряд $\left\{\mathrm{У} / \mathrm{H}_{2} \mathrm{O}_{2}\right\}>\left\{\mathrm{H}_{2} \mathrm{O}_{2}\right\}>\{У \Phi\}$. Установлено, что оптимальные условия окисления $\mathrm{As}$ (III) реализуются при рН 5,8-9, при стехиометрическом молярном соотношении $\left[\mathrm{H}_{2} \mathrm{O}_{2}\right]-\left[\mathrm{As}^{3+}\right]$, равном 1. При этом дозы УФ излучения, необходимые для окисления 90 \% As(III), составили 150-380 мДж/ $\mathrm{cm}^{2}$.

Предложен смешанный ион-радикальный механизм фотохимического окисления мышьяка (III) с использованием УФ-С излучения $\mathrm{KrCl}$-эксилампы в присутствии пероксида водорода, заключающийся в окислении мышьяка АФК преимущественно гидроксильными и супероксидными радикалами и последующем окислении интермедиатов растворенным кислородом. При этом лимитирую-

\section{СПИСОК ЛИТЕРАТУРЫ}

1. Smedley P.L., Kinniburgh D.G. A review of the source, behavior and distribution of arsenic in natural waters // Applied Geochemistry. - 2002. - V. 17. - № 5. - P. 517-568.

2. Multiple inorganic toxic substances contaminating the groundwater of Myingyan Township, Myanmar: Arsenic, manganese, fluoride, iron, and uranium / T. Bacquart, S. Frisbie, E. Mitchell, L. Grigg, C. Cole, C. Small, B. Sarkar // Science of the Total Environment. - 2015. - V. 517. - P. 232-245.

3. Current status of arsenic contamination in drinking water and treatment practice in some rural areas of west Bengal, India J.N. Bhakta, S. Ranaa, J. Janaa, S.K. Baga, S. Lahiria, B.B. Janaa, F. Panningc, L. Fechterd // Journal of Water Chemistry and Technology. - 2016. - V. 38. - № 6. - P. 366-373.

4. Arsenic speciation and uranium concentrations in drinking water supply wells in Northern Greece: Correlations with redox indicative parameters and implications for groundwater treatment I.A. Katsoyiannis, S.J. Hug, A. Ammann, A. Zikoudi, C. Hatziliontos // Science of the Total Environment. - 2007. - V. 383. P. 128-140.

5. Avoiding high concentrations of arsenic, manganese and salinity in deep tubewells in Munshiganj District, Bangladesh / S.J. Hug, D. Gaertnera, L.C. Robertsa, M. Schirmera, T. Ruettimanna, T.M. Rosenbergb, A.B.M. Badruzzamanc, A.M. Ali // Applied Geochemistry. - 2011. - V. 26. - P. 1077-1085.

6. Prevalence of high arsenic concentration in Darbhanga district of Bihar: Health assessment / A. Abhinav, S. Navin, A. Kumar, R. Kumar, M. Ali, S.K. Verma, A.K. Ghosh // Journal of Environmental and Analytical Toxicology. - 2016. - V. 6. - № 6. P. 1-7.

7. Mohan D., Pittman Ch.U. Jr. Arsenic removal from water/wastewater using adsorbents - a critical review // Journal of Hazardous Materials. - 2007. - V. 142. - P. 1-53.

8. Коваленко К.А., Бочкарев Г.Р., Пушкарева Г.И. Марганцевые руды как перспективный сорбент для удаления соединений мышьяка из подземных вод // Вода: Химия и экология. 2013. - № 10. - C. 80-84.

9. Arsenic (III) remediation from contaminated water by oxidation and $\mathrm{Fe} / \mathrm{Al}$ co-precipitation / W. Zhang, P. Singh, T.B. Issa, W. Zhang // Journal of Water Resource and Protection. 2011. - № 3. - P. 655-660.

10. Removal of arsenic (III) and arsenic (V) from aqueous medium using chitosan-coated biosorbent / V.M. Boddu, K. Abburi, J.L. Talbott, E.D. Smith, R. Haasch // Water Research. - 2008. V. $42 .-$ P. $633-642$.

11. Iron oxide-modified nanoporous geopolymers for arsenic removal from ground water / D. Medpelli, R. Sandoval, L. Sherrill, K. Hristovski, D.-K. Seo // Resource-Efficient Technologies. 2015. - V. 1. - № 1. - P. 19-27. щей стадией всего процесса является окисление интермедиата $\mathrm{As}(\mathrm{IV})$.

Выявлено, что $\mathrm{HCO}_{3}{ }^{-}$в концентрациях до $130 \mathrm{мг} /$ л, наиболее характерные анионы природных «водных матриц», оказывают ингибирующее действие на протекание реакций в комбинированном процессе.

Полученные результаты свидетельствуют 0 принципиальной возможности реализации процесса полного окисления As(III) с использованием энергоэффективного источника оптического излучения $\mathrm{KrCl}$-эксилампы (222 нм) для очистки мышьяксодержащих природных и сточных вод.

Работа выполнена в рамках государственного задания БИП СО РАН № 007-00207-18-00.

12. Eary L.E., Schramke J.A. Rates of Inorganic Oxidation Reactions Involving Dissolved Oxygen / Chemical Modeling of Aqueous Systems II. American Chemical Society Symposium Series, 1990. V. 416. - № 30. - P. 379-396.

13. Pettine M., Companella L., Millelero F.J. Arsenite oxidation by $\mathrm{H}_{2} \mathrm{O}_{2}$ in aqueous solutions // Geochimica et Cosmochimica Acta. 1999. - V. 63. - № 18. - P. 2727-2735.

14. Deng Y., Zhao R. Advanced oxidation processes (AOPs) in wastewater treatment // Current Pollution Reports. - 2015. - V. 1. - P. 167-176.

15. Hug S.J., Leupin 0. Iron-catalyzed oxidation of arsenic (III) by oxygen and by hydrogen peroxide: $\mathrm{pH}$-dependent formation of oxidants in the Fenton reaction // Environmental Science and Technology. - 2003. - V. 37. - P. 2734-2742.

16. Katsoyiannis I.A., Ruettiman T., Hug S.J. Ph Dependence of Fenton Reagent generation and As (III) oxidation and removal by corrosion of Zero valent iron in aerated water // Environmental Science and Technology. - 2008. - V. 42. - P. 7424-7430.

17. Comparing the efficacy of VUV and UVC $/ \mathrm{S}_{2} \mathrm{O}_{8}{ }^{2-}$ advanced oxidation processes for degradation and mineralization of cyanide in wastewater / G. Moussavi, M. Pourakbar, E. Aghayani, M. Mahdavianpour, S. Shekoohyian // Chemical Engineering Journal. - 2016. - V. 294. - P. 273-280.

18. Ультрафиолетовые и вакуумно-ультрафиолетовые эксилампы: физика, техника и применения / А.М. Бойченко, М.И. Ломаев, А.Н. Панченко, Э.А. Соснин, В.Ф. Тарасенко. - Томск: Изд-во STT, 2011. - 512 с.

19. Sosnin E.A., Oppenländer T., Tarasenko V.F. Applications of capacitive and barrier discharge excilamps in photoscience // Journal of Photochemistry and Photobiology C: Photochemistry Reviews. - 2006. - № 7. - P. 145-163.

20. Canonica S., Meunier L., Von Gunten U. Phototransformation of selected pharmaceuticals during UV treatment of drinking water // Water Research. - 2008. - V. 42. - P. 121-128.

21. Комбинированный метод деградации хлорфенолов / Г.Г. Матафонова, В.Б. Батоев, Э.А. Соснин, N. Christofi // Химия в интересах устойчивого развития. - 2008. - Т. 16. - С. 191-197.

22. Матафонова Г.Г., Батоев В.Б. Деструкция карбамазепина в воде ультрафиолетовым излучением эксилампы // Вода: химия и экология. - 2013. - № 9. - С. 87-92.

23. Monitoring of $\mathrm{TiO}_{2}$-catalytic UV-LED photo-oxidation of cyanide contained in mine wastewater and leachate / S.H. Kim, S.W. Lee, G.M. Lee, B.T. Lee, S.T. Yun, S.0. Kim // Chemosphere. 2016. - V. 143. - № 1. - P. 106-114.

24. Photocatalytic oxidation of arsenic (III) evidence of hydroxyl radicals / P.K. Dutta, S.O. Pehkonen, V.K. Sharma, A.K. Ray // Environmental Science and Technology. - 2005. - V. 39. - P. 1827-1834.

25. ГОСТ 4152-89. Вода питьевая. Метод определения массовой концентрации мышьяка. - Введ. 1991-01-01. - М.: Госстандарт России: Изд-во стандартов, 1991. - С. 473-479. 
26. Christensen J., Linden K.G. How particles affect UV lighting the UV disinfection of unfiltered drinking water // Journal of the American Water Works Association. - 2003. - № 95. P. $179-189$.

27. Arsenite oxidation by ultrasound combined with ultraviolet in aqueous solution / S. Lee, M. Cui, S. Na, J. Khim // Japanese Journal of Applied Physics. - 2012. - V. 51. - 07GD15.

28. Нормативы качества воды водных объектов рыбохозяйственного значения. Приказ Минсельхоза России, 13 декабря 2016, № 552 .

29. Предельно допустимые концентрации (ПДК) химических веществ в воде водных объектов хозяйственно-питьевого и культурно-бытового водопользования. Гигиенические нормативы ГН 2.1.5.1315-03. (с изм. на 13.07.2017 г.). - М.: Электронный фонд правовой и нормативно-технической документации. URL: http://docs.cntd.ru/document/901862249 (дата обращения 15.04.2019).

30. Ryu J., Choi W. Photocatalytic oxidation of Arsenite on $\mathrm{TiO}_{2}$ : understanding the controversial oxidation mechanism involving superoxides and effect of alternative electron acceptors // Environmental Science and Technology. - 2006. - V.40. - P. 7034-7039.

31. Baxendale J.H., Wilson J.A. The photolysis if hydrogen peroxide at high light intensities // Transactions of the Faraday Society. 1957. - V. 53. - № 411. - P. 344-356.

32. Glaze W.H., Kang J.-W., Chapin D.H. The chemistry of water treatment processes involving ozone, hydrogen peroxide and ul- traviolet radiation // Ozone: Science and engineering. - 1987. V. 9. - P. 335-352.

33. Klaning U.K., Bielski B.H.J., Sehested K. Arsenic (IV). A pulseradiolysis study // Inorganic Chemistry. - 1989. - V. 28. P. 2717-2724.

34. Critical review of rate constants for reactions of hydrated electrons, hydrogen atoms and hydroxyl radicals in aqueous solution / G.V. Buxton, C.L. Greenstock, W.P. Helman, A.B. Ross // Journal of Physical and Chemical Reference Data. - 1988. V. 17. - P. 513-886.

35. Effect of $\mathrm{HO}$, and $\mathrm{SO}_{4}{ }^{-{ }^{-}}$and $\mathrm{CO}_{3}{ }^{-{ }^{-}} / \mathrm{HCO}_{3}{ }_{3}$ radicals on the photodegradation of the herbicide amitrole by UV radiation in aqueous solution / F. Orellana-Garcia, M.A. Álvarez, M.V. López-Ramón, J. Rivera-Utrilla, M. Sánchez-Polo // Chemical Engineering Journal. - 2015. - V. 267. - P. 182-190.

36. Lutze H.V., Kerlin N., Schmidt T.C. Sulfate radical-based water treatment in presence of chloride: Formation of chlorate, interconversion of sulfate radicals into hydroxyl radicals and influence of bicarbonate // Water Research. - 2015. - V. 72. P. 349-360.

37. Sokolova I.V., Tchaikovskaya 0.N. Fluorescence and photochemical properties of humic acids // Atmospheric and Oceanic optics. - 2006. - V. 19. - № 02-03. - P. 220-222.

Поступила 29.04.2019 2.

\section{Информация об авторах}

Цыбикова Б.А., кандидат технических наук, научный сотрудник лаборатории инженерной экологии Байкальского института природопользования СО РАН.

Батоева A.A., доктор технических наук, доцент, заведующий лабораторией инженерной экологии Байкальского института природопользования СО РАН. 
UDC 544.526.2

\title{
EXCILAMPS AS A PERSPECTIVE SOURCE OF UV RADIATION FOR PHOTOCHEMICAL DETOXIFICATION OF ARSENIC CONTAINING WATERS
}

\author{
Belegma A. Tsybikova', \\ belegmats@mail.ru \\ Agniya A. Batoeva', \\ abat@binm.ru \\ Baikal Institute of Nature Management SB RAS, \\ 6, Sakhyanova street, Ulan-Ude, 670047, Russia.
}

The relevance of the research is determined by the need to solve the problem of toxic substances, including arsenic compounds, entry into water bodies, by searching for and developing effective photochemical methods for cleaning and neutralizing arsenic-containing solutions using environmentally friendly oxidants and modern mercury-free sources of UV-radiation.

The main aim of the research is to study the possibilities of using excilamps for neutralizing As-containing waters and to establish the regularities of photochemical activation of arsenic oxidation by hydrogen peroxide using a mercury-free source of quasi-monochromatic radiation from the $\mathrm{KrCl}$ excilamp.

Objects: model aqueous solutions of arsenic (III) with a concentration of 2,7 $\mu \mathrm{M}(\sim 0,2 \mathrm{mg} / \mathrm{L})$, prepared on distilled water (UEP=2 $\mu \mathrm{S} / \mathrm{cm}, \mathrm{pH} 5,8)$ and tap water.

Methods: spectrophotometric determination of arsenic (III) ions; pH control by potentiometric method, shooting of electronic absorption spectra of solutions of reacting substances; determination of absorbed emission power of the excilamp by the method of chemical actinometry with atrazine; evaluation of doses of UV-radiation.

Results. The authors have established the principal possibility of using the mercury-free source of quasi-monochromatic radiation of the $\mathrm{KrCl}$ excilamp $(222 \mathrm{~nm})$ for photochemical oxidation of $\mathrm{As}(I I I)$ by the oxidation system $\left\{\mathrm{UV} / \mathrm{H}_{2} \mathrm{O}_{2}\right\}$. They studied the main regularities of photochemical oxidation of arsenic (III) using the mercury-free UV source $\mathrm{KrCl}$-excilamp (222 nm) and carried out the comparative studies of the efficiency of direct photolysis and combined oxidation of arsenic (III) in aqueous solution with ultraviolet radiation of the $\mathrm{KrCl}$ excilamp in the presence of hydrogen peroxide. According to the efficiency of As (III) oxidation the considered oxidation systems may be arranged as $\left\{U V / H_{2} \mathrm{O}_{2}\right\}>\left\{\mathrm{H}_{2} \mathrm{O}_{2}\right\}>\{U V\}$. At the combined treatment of $\left\{U V / \mathrm{H}_{2} \mathrm{O}_{2}\right\}$ the significant intensification of oxidation is observed, after 10 minutes the residual arsenic concentration in the solution is below the MPC $(0,01 \mathrm{mg} / \mathrm{l})$. The paper considers as well the effect of hydrocarbonate ions, being the most characteristic anions of natural "water matrices», on the oxidation rate of As(III). The authors proposed the ion-radical mechanism of arsenic photochemical oxidation in a combined oxidation system $\left\{U V / \mathrm{H}_{2} \mathrm{O}_{2}\right\}$ using the UV-radiation of the $\mathrm{KrCl}$ excilamp.

Key words:

Arsenic, hydrogen peroxide, mercury-free source of UV radiation, KrCl-excilamp, hydrocarbonates, combined oxidation processes.

The research was carried out within the State Task of Baikal Institute of Nature Management SB RAS no. 007-00207-18-00.

\section{REFERENCES}

1. Smedley P.L., Kinniburgh D.G. A review of the source, behavior and distribution of arsenic in natural waters. Applied Geochemistry, 2002, vol. 17, no. 5, pp. 517-568.

2. Bacquart T., Frisbie S., Mitchell E., Grigg L., Cole C., Small C., Sarkar B. Multiple inorganic toxic substances contaminating the groundwater of Myingyan Township, Myanmar: Arsenic, manganese, fluoride, iron, and uranium. Science of the Total Environment, 2015, vol. 517, pp. 232-245.

3. Bhakta J.N., Ranaa S., Janaa J., Baga S.K., Lahiria S., Janaa B.B., Panningc F., Fechterd L. Current status of arsenic contamination in drinking water and treatment practice in some rural areas of west Bengal, India. Journal of Water Chemistry and Technology, 2016, vol. 38, no. 6, pp. 366-373.

4. Katsoyiannis I.A., Hug S.J., Ammann A., Zikoudi A., Hatziliontos C. Arsenic speciation and uranium concentrations in drinking water supply wells in Northern Greece: Correlations with redox indicative parameters and implications for groundwater treatment. Science of the Total Environment, 2007, vol. 383, pp. $128-140$.

5. Hug S.J., Gaertnera D., Robertsa L.C., Schirmera M., Ruettimanna T., Rosenbergb T.M., Badruzzamanc A.B.M., Ali A.M. Avoiding high concentrations of arsenic, manganese and salinity in deep tubewells in Munshiganj District, Bangladesh. Applied Geochemistry, 2011, vol. 26, pp. 1077-1085.

6. Abhinav A., Navin S., Kumar A., Kumar R., Ali M., Verma S.K., Ghosh A.K. Prevalence of high arsenic concentration in Darbhanga district of Bihar: Health assessment. Journal of Environmental and Analytical Toxicology, 2016, vol. 6, no. 6, pp. 1-7.

7. Mohan D., Pittman Ch.U. Jr. Arsenic removal from water/wastewater using adsorbents - a critical review. Journal of Hazardous Materials, 2007, vol. 142, pp. 1-53.

8. Kovalenko K.A., Botchkarev G.R., Pushkareva G.I. Manganese ores as a promising sorbent for the removal of arsenic compounds from groundwater. Water: Chemistry and Ecology, 2013, no. 10, pp. 80-84. In Rus.

9. Zhang W., Singh P., Issa T.B., Zhang W. Arsenic (III) remediation from contaminated water by oxidation and $\mathrm{Fe} / \mathrm{Al}$ co-precipitation. Journal of Water Resource and Protection, 2011, no. 3, pp. $655-660$.

10. Boddu V.M., Abburi K., Talbott J.L., Smith E.D., Haasch R. Removal of arsenic (III) and arsenic (V) from aqueous medium using chitosan-coated biosorbent. Water Research, 2008, vol. 42, pp. 633-642.

11. Medpelli D., Sandoval R., Sherrill L., Hristovski K., Seo D.-K. Iron oxide-modified nanoporous geopolymers for arsenic removal 
from ground water. Resource-Efficient Technologies, 2015, vol. 1, no. 1, pp. 19-27.

12. Eary L.E., Schramke J.A. Rates of Inorganic Oxidation Reactions Involving Dissolved Oxygen. Chemical Modeling of Aqueous Systems II. American Chemical Society Symposium Series, 1990, vol. 416, no. 30, pp. 379-396.

13. Pettine M., Companella L., Millelero F.J. Arsenite oxidation by $\mathrm{H}_{2} \mathrm{O}_{2}$ in aqueous solutions. Geochimica et Cosmochimica Acta, 1999, vol. 63, no. 18, pp. 2727-2735.

14. Deng Y., Zhao R. Advanced oxidation processes (AOPs) in wastewater treatment. Current Pollution Reports, 2015, vol. 1, pp.167-176.

15. Hug S.J., Leupin 0. Iron-catalyzed oxidation of arsenic (III) by oxygen and by hydrogen peroxide: $\mathrm{pH}-$ dependent formation of oxidants in the Fenton reaction. Environmental Science and Technology, 2003, vol. 37, pp. 2734-2742.

16. Katsoyiannis I.A., Ruettiman T., Hug S.J. Ph Dependence of Fenton Reagent generation and As (III) oxidation and removal by corrosion of Zero valent iron in aerated water. Environmental Science and Technology, 2008, vol. 42, pp. 7424-7430.

17. Moussavi G., Pourakbar M., Aghayani E., Mahdavianpour M., Shekoohyian S. Comparing the efficacy of VUV and UVC $/ \mathrm{S}_{2} \mathrm{O}_{8}{ }^{2-}$ advanced oxidation processes for degradation and mineralization of cyanide in wastewater. Chemical Engineering Journal, 2016, vol. 294, pp. 273-280.

18. Boychenko A.M., Lomaev M.I., Panchenko A.N., Sosnin E.A., Tarasenko V.F. Ultrafioletovye i vakuumno-ultrafioletovye eksilampy: fizika, tekhnika i primeneniya [Ultraviolet and vacuum-ultraviolet excilamps: physics, engineering and applications]. Tomsk, STT Publ., 2011. $512 \mathrm{p}$.

19. Sosnin E.A., Oppenländer T., Tarasenko V.F. Applications of capacitive and barrier discharge excilamps in photoscience. Journal of Photochemistry and Photobiology C: Photochemistry Reviews, 2006, no. 7, pp. 145-163.

20. Canonica S., Meunier L., Von Gunten U. Phototransformation of selected pharmaceuticals during UV treatment of drinking water. Water Research, 2008, vol. 42, pp. 121-128.

21. Matafonova G.G., Batoyev V.B., Sosnin E.A., Christofi N. Combined method of degradation of chlorophenols. Chemistry for $\mathrm{Su}$ stainable Development, 2008, vol. 16, pp. 191-197. In Rus.

22. Matafonova G.G., Batoyev V.B. Destruction of carbamazepine in water by ultraviolet radiation of the excilamps. Water: Chemistry and Ecology, 2013, no. 9, pp. 87-92. In Rus.

23. Kim S.H., Lee S.W., Lee G.M., Lee B.T., Yun S.T., Kim S.O. Monitoring of $\mathrm{TiO}_{2}$-catalytic UV-LED photo-oxidation of cyanide contained in mine wastewater and leachate. Chemosphere, 2016, vol. 143, no. 1, pp. 106-114.

24. Dutta P.K., Pehkonen S.O., Sharma V.K., Ray A.K. Photocatalytic oxidation of arsenic (III) evidence of hydroxyl radicals. Environmental Science and Technology, 2005, vol. 39, pp. $1827-1834$.

25. GOST 4152-89. Voda pityevaya. Metod opredeleniya massovoy kontsentratsii myshyaka [State Standard 4152-89. Drinking water. Method for determination of arsenic mass concentration]. Moscow, Standartinform Publ, 1991. pp. 473-479.
26. Christensen J., Linden K.G. How particles affect UV lighting the UV disinfection of unfiltered drinking water. Journal of the American Water Works Association, 2003, no. 95, pp. 179-189.

27. Lee S., Cui M., Na S., Khim J. Arsenite oxidation by ultrasound combined with ultraviolet in aqueous solution. Japanese Journal of Applied Physics, 2012, vol. 51, 07GD15.

28. Normativy kachestva vody vodnykh obyektov rybokhozyaystvennogo znacheniya [Water quality standards for water bodies of fisheries value. Order of the Ministry of Agriculture of Russia, December 13, 2016, № 552.]. Order of the Ministry of Agriculture of Russia, December 13, 2016. no. 552.

29. Predelno dopustimye kontsentratsii (PDK) khimicheskikh veshchestv v vode vodnykh obyektov khozyaystvenno-pitevogo $i \mathrm{ku}$ lturno-bytovogo vodopolzovaniya [Maximum permissible concentration (MPC) of chemical substances in water of water objects of domestic and drinking water and cultural and domestic water use]. Hygienic standards GN 2.1.5.1315-03 (as amended on 07/13/2017) [Electronic resource]. Moscow, Electronic fund of legal and regulatory technical documentation. Available at: http://docs.cntd.ru/document/901862249 (accessed 15 April 2019).

30. Ryu J., Choi W. Photocatalytic oxidation of Arsenite on $\mathrm{TiO}_{2}$ : understanding the controversial oxidation mechanism involving superoxides and effect of alternative electron acceptors. Environmental Science and Technology, 2006, vol. 40, pp. 7034-7039.

31. Baxendale J.H., Wilson J.A. The photolysis if hydrogen peroxide at high light intensities. Transactions of the Faraday Society, 1957, vol. 53, no. 411, pp. 344-356.

32. Glaze W.H., Kang J.-W., Chapin D.H. The chemistry of water treatment processes involving ozone, hydrogen peroxide and ultraviolet radiation. Ozone: Science and engineering, 1987, vol. 9, pp. $335-352$.

33. Klaning U.K., Bielski B.H.J., Sehested K. Arsenic (IV). A pulseradiolysis study. Inorganic Chemistry, 1989, vol. 28, pp. 2717-2724.

34. Buxton G.V., Greenstock C.L., Helman W.P., Ross A.B. Critical review of rate constants for reactions of hydrated electrons, hydrogen atoms and hydroxyl radicals in aqueous solution. Journal of Physical and Chemical Reference Data, 1988, vol. 17, pp. 513-886.

35. Orellana-Garcia F., Álvarez M.A., López-Ramón M.V., RiveraUtrilla J., Sánchez-Polo M. Effect of $\mathrm{HO}^{-}$, and $\mathrm{SO}_{4}{ }^{-{ }^{-}}$and $\mathrm{CO}_{3}{ }^{--} / \mathrm{HCO}_{3}{ }^{-}$radicals on the photodegradation of the herbicide amitrole by UV radiation in aqueous solution. Chemical Engineering Journal, 2015, vol. 267, pp. 182-190.

36. Lutze H.V., Kerlin N., Schmidt T.C. Sulfate radical-based water treatment in presence of chloride: Formation of chlorate, interconversion of sulfate radicals into hydroxyl radicals and influence of bicarbonate. Water Research, 2015, vol. 72, pp. 349-360.

37. Sokolova I.V., Tchaikovskaya 0.N. Fluorescence and photochemical properties of humic acids. Atmospheric and Oceanic optics, 2006, vol. 19, no. 02-03, pp. 220-222.

Received: 29 April 2019.

\section{Information about the authors}

Belegma A. Tsybikova, Cand. Sc., researcher, Baikal Institute of Nature Management SB RAS.

Agniya A. Batoeva, Dr. Sc., associate professor, head of the Laboratory, Baikal Institute of Nature Management SB RAS. 